\title{
Building a Complete Surface Model from Sparse Data Using Statistical Shape Models: Application to Computer Assisted Knee Surgery
}

\author{
Markus Fleute and Stéphane Lavallée, $\mathrm{PhD}$ \\ TIMC - IAB, Faculté de Médecine de Grenoble, 38706 La Tronche Cedex FRANCE \\ markus.fleute@imag.fr
}

\begin{abstract}
This paper addresses the problem of extrapolating very few range data to obtain a complete surface representation of an antomical structure. A new method that uses statistical shape models is proposed and its application to modeling a few points manually digitized on the femoral surface is detailed, in order to improve visualization of a system developped by TIMC laboratory for computer assisted anterior cruciate ligament (ACL) reconstruction. The model is built from a population of 11 femur specimen digitized manually. Data sets are registered together using an elastic registration method of Szeliski and Lavallée based on octree-splines. Principal Components Analysis (PCA) is performed on a field of surface deformation vectors. Fitting this statistical model to a few points is performed by non-linear optimisation. Results are presented for both simulated and real data. The method is very flexible and can be applied to any structures for which the shape is stable.
\end{abstract}

\section{Introduction}

A system for computer assisted anterior cruciate ligament (ACL) reconstruction has been developped by TIMC laboratory since 1993. Details about the technique and its clinical validation can be found in [DLO+95, DLJ $+95, J L D 98]$.

The system uses only intra operative data obtained with an optical localizer (Optotrak, Northern Digital, Toronto), without requiring additional images such as X-rays, CT or MRI. The system enables the surgeon to digitize 3D points interactively, to track relative bone motion, and to locate the pose of surgical tools in real time. Rigid bodies made of infra-red LEDs are attached to the bones, the pointers, and the tools (Fig.1(a)). In the current version of the system, clouds of digitized points are approximated by spline patches. Those data enable the surgeon to navigate in the computer screen in real-time with any surgical tool equipped with an optical rigid body, and to compare the position of the tool with an optimal position of a graft defined by 2 points $F$ and $T$ on the femoral and tibial surfaces. Currently, the system enables the surgeon to minimize the anisometry of the graft by displaying anisometry maps [DLO $\left.{ }^{+} 95\right]$, whilst avoiding impingement between the graft and the femoral notch [JLD98].

Fig.2(a) shows the Graphical User Interface of the current system for Computer Assisted ACL surgery including anisometry maps, a $3 \mathrm{D}$ view with spline 


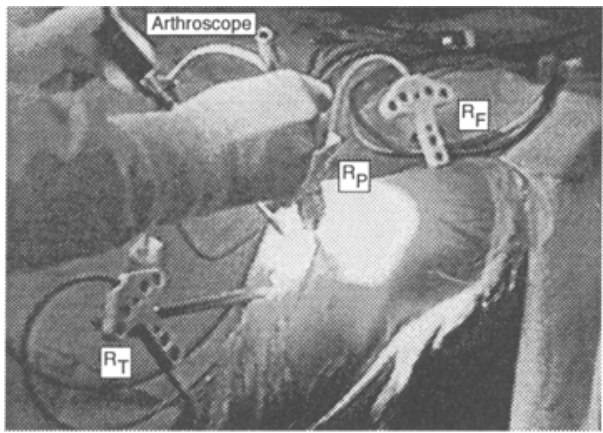

(a)

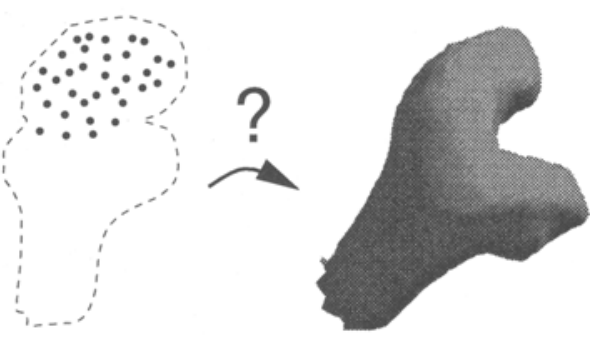

(b)

Fig. 1. (a) Surface digitizing during surgery. (b) Problem: How to extrapolate a few data points to a complete femur?

surfaces and a model of the graft envelope. Since only a small surface area has been digitized, it is very difficult to recognize the actual pose of the tibia and the femur only from the small surfaces patches generated by the bicubic splines. In order to provide the surgeon with a more complete and realistic view of the scene it is desirable to have a visualization of the whole femur (respectively tibia) as shown in Fig.2(b) (only the femur is visualized).

As illustrated in Fig.1(b), the problem addressed in this paper is to recover the complete shape of the bones from the few available data points. The objectives of this work are quadruple:

- Visualization of a complete anatomical model, including anatomical landmarks, for better orientation.

- Post-operative referencing of clinical results in the model coordinate system

- Shape-based interpolation in the area of digitized points

- Extrapolation coherent with the anatomy in the areas far from the digitized points

\section{Related Work}

The literature of computer vision and deformable models proposes a large variety of methods for building a surface model from range data. Overviews can be found in [BV91,MT96]. However, most of methods use only local constraints such as smoothness or they fit global shapes such as planes, cylinders or superquadrics which are not apropriate for our purpose, since the resulting shapes are not coherent with the anatomy if only a few data points locally distributed are used. It is therefore necessary to incorporate global a priori knowledge contained in statistical models, using Fourier representations [SkBG96], modal analysis performed directly [PS91] or based on features such as crest lines [STA96], or 


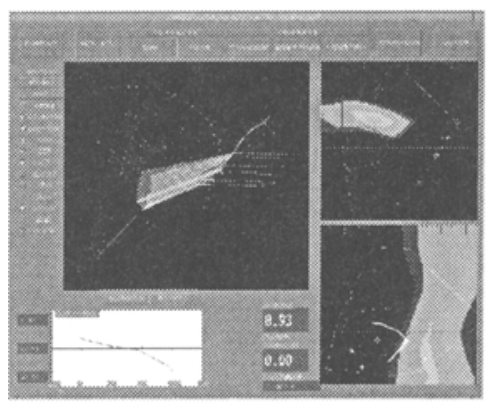

(a)

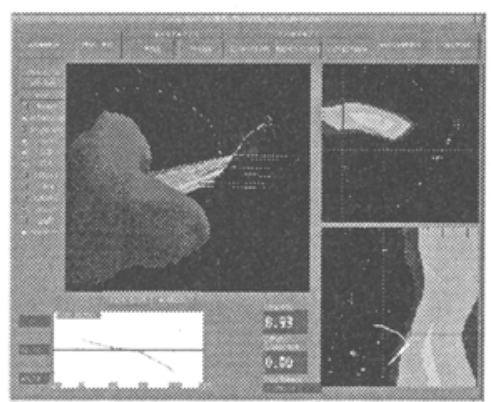

(b)

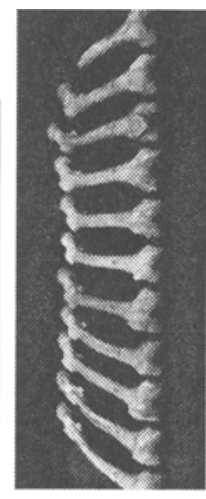

(c)

Fig. 2. (a) GUI for computer assisted ACL reconstruction (b) GUI using a complete model of the femur instead of a bicubic spline in the $3 \mathrm{D}$ view window : the global orientation and anatomy of the knee can be understood. (c) 11 right femurs of specimen used to build the model

Point distribution Models (PDM) proposed by Cootes and Taylor [CTCG95]. Our method is based on the latter approach.

A PDM is a deformable model built from the statistical analysis of examples of the object being modelled. Given a collection of $N$ 3D training shapes of an object, the Cartesian coordinates of $M$ landmark points are recorded for each image. Each training example is represented by a vector $\mathbf{m}=\left(x_{1}, y_{1}, z_{1}, \ldots, x_{M}, y_{M}, z_{M}\right)$. After aligning of the training shapes the pointwise mean shape $\overline{\mathbf{m}}=\frac{1}{N} \sum_{i=1}^{N} \mathbf{m}_{i}$ is then calculated. Modes of variation are found using Principal Component Analysis (PCA) on the deviations of examples from the mean. These modes are represented by $3 M$ orthonormal eigenvectors $\mathbf{e}_{i}$. A new instance of the shape is generated by adding linear combinations of the $t$ most significant variation vectors to the mean shape: $\mathbf{m}=\overline{\mathbf{m}}+\sum_{i=1}^{t} w_{i} \mathbf{e}_{i}$ where $w_{i}$ is the weighting for the $i^{t h}$ variation vector. By ensuring $t<3 M$, only the important deformations are extracted, discarding training data noise, and thus object shape and variation can be captured compactly. A key requirement for building such a model is the collection of several sets with corresponding landmarks from training images. This is presented in the following section.

\section{Building a statistical shape model}

Acquiring the Training Shapes: A set of 11 dry femurs of specimen (Fig.2(c)) was digitized manually using a 3 -D optical localizer, resulting in 11 non-organized point sets. Ten of the femurs have been digitized with approximately 1500 points randomly distributed on the bone surface. The eleventh case was considered separately as a template and was digitized with a superior density in a larger 
area of the femur, resulting in approximately 6000 points. To obtain one surface representation of this template, an algorithm proposed by Hoppe [HDDW92] was used to build facets from the cloud of points, and then the result was simplified using the decimation method proposed in [CCMS97]. The result is a triangular mesh $T M_{r e f}$ of approximative 1500 vertices.

Aligning and matching the training shapes using Octree Splines: Now each of the 10 point sets has to be matched to the template mesh in such a way that each vertex of the template mesh $T M_{\text {ref }}$ is mapped to its anatomically corresponding point on the femur represented by the set of points. We propose to use a multiresolution approach proposed by Szeliski and Lavallée, based on octree-splines [SL96]. The method performs a least squares minimization of the distances between a sparse and unorganized set of points and a dense set of points used to build a 3D octree-spline distance map [LSB91]. In our case, the dense set of points is obtained by resampling each facet of the template triangular mesh $T M_{\text {ref }}$ up to 10,000 points. Fig.3(a) shows the octree containing the two shapes to be registered after rigid registration (top) and after non rigid registration (bottom).

The result of the octree-spline based registration technique is a smooth volumetric transform $T$ that maps every point $P$ of the data space to a point $M=T(P)$ of the model space. Reciprocally, it is possible to inverse this transformation. Given a point $M$ of the template mesh, an iterative search is performed to find the point $P=T^{-1}(M)$ such that $\|M-T(P)\|<\epsilon$. By this process, each point $M_{i}$ of the template mesh is assigned to a data point $P_{i}$ for each data set $\mathrm{j}$ of the $\mathrm{N}$ data spaces. Note that the points $P_{i}$ were not in the data sets initially, but they were implicitly interpolated using the octree-spline deformation.

To avoid having the template mesh as the reference while registering the training shapes, all objects have to be registered to the final mean shape. This is done using an iterative algorithm: At first all training shapes are matched to the template mesh as described above. After calculating the mean shape, now all training shapes are matched to this current mean. This process is repeated until convergence occurs. The displacement vectors $\mathbf{d}_{m_{i}}=\mathbf{m}_{i}-\overline{\mathbf{m}}$ describe the mapping between the mean shape and each of the training objects.

Principal Component Analysis: Hence one can apply a Principal Component Analysis to the data which results in finding the eigenvectors of the covariance matrix $\mathbf{C}=\frac{1}{N} \sum_{i=1}^{N} \mathbf{d}_{m_{i}} \mathbf{d}_{m_{i}}^{T}$ of dimension $3 M \times 3 M$ which can be calculated from the displacement vectors. If $N \geq 3 M$ one obtains $3 M-1$ nonzero eigenvectors. If $N<3 M$ it can be shown that the eigenvectors of the covariance matrix can be calculated from a smaller $N \times N$ matrix derived from the same data [CTCG95]. In this case there are only $N-1$ nonzero eigenvectors. Because the eigenvector calculation time goes as the cube of the size of the matrix, this can give substantial time savings, as $3 M$ may be in the range of several thousands, while $N$ normally is much smaller. As $\mathbf{C}$ is real and symmetric effective Jacobi transformations can be applied to obtain the eigenvectors [PFTV92]. 

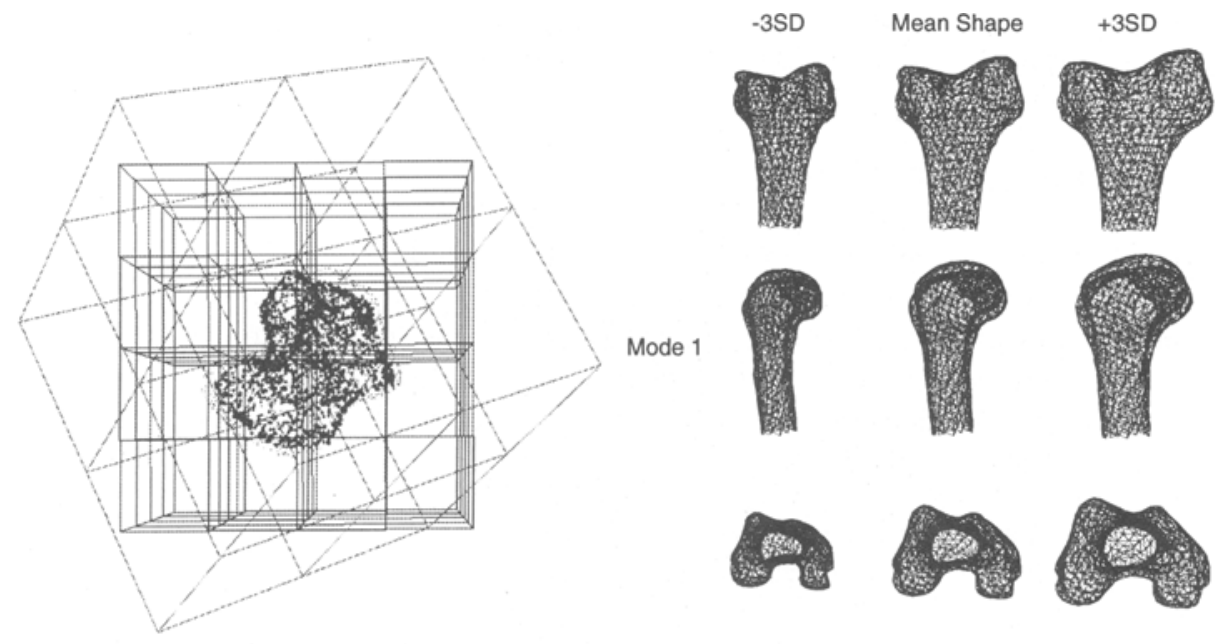

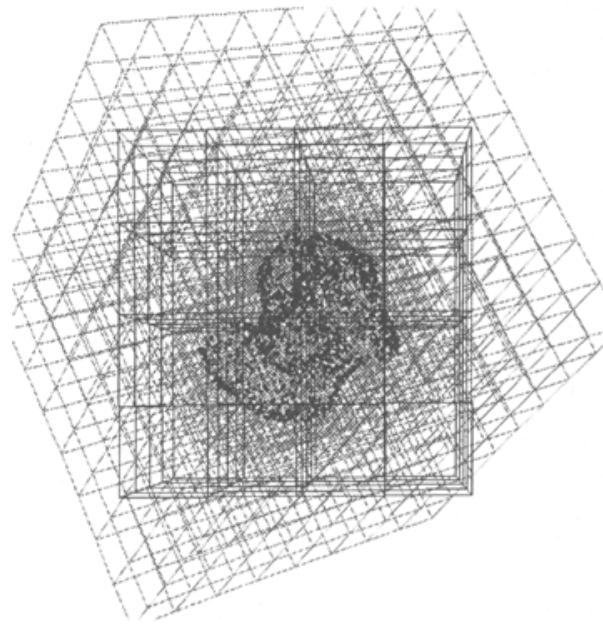

(a)

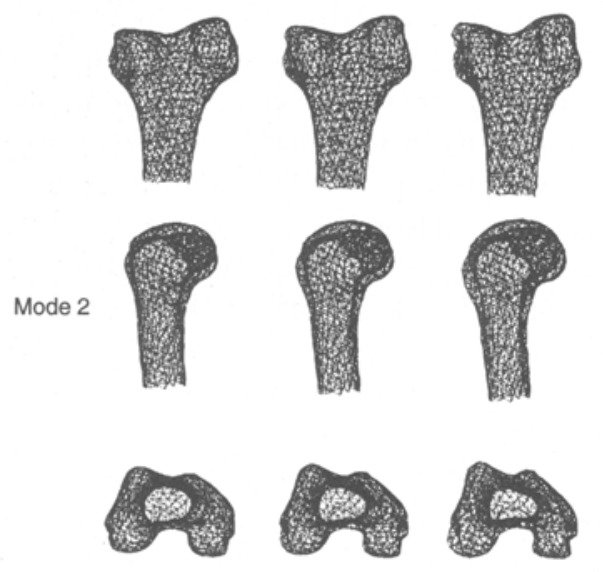

(b)

Fig. 3. (a) Hierarchical volumetric deformation of a low density training set with a high density template mesh, using Octree Splines. Up: after rigid alignment. Bottom: after elastic registration. (b) Applying 3 standard deviations of the first and second deformation modes on the mean shape 
The mean surface and the $t$ principal modes obtained by PCA constitute the statistically-based shape model.

\section{$4 \quad$ Fitting the model}

To recover the whole surface of the object from a new set made of a few sparse data points it is necessary to find simultaneously the rigid transformation (rotation $\mathbf{R}$, translation $\mathbf{T}$ ) between the data and the model and the decomposition of the $t$ preserved eigenvectors in such a way that the distances between the data points and the model are minimized. The objective function to be minimized is defined as follows:

$$
f=\sum_{i=1}^{D} \min _{1 \leq j \leq M}\left\|\mathbf{d}_{i}-\mathbf{m}_{j}\right\|^{2}
$$

with $\mathbf{m}_{j}=\mathbf{R}\left(\overline{\mathbf{m}}+\sum_{i=1}^{t} w_{i} \mathbf{e}_{i}\right)+\mathbf{T}, D$ the number of data points, and $\mathbf{d}_{i}$ the $i^{t h}$ data point. The computationally most expensive step in the registration process is finding the closest point in the model to each data point. The computational complexity evaluating $f$ is $\mathbf{O}(D M)$ using exhaustive search. It is not interesting to compute a 3-D distance map here because the model is deformed at each iteration. Therefore $k$-dimensional binary trees ( $k$ - $d$ trees, in this case $k=3$ ) are used to speed up the computation [FBF77]. A k-d tree can be constructed in $\mathbf{O}(M \log (M))$. Searching the closest point in the tree to the given data points can then be performed in $\mathbf{O}(D \log (M))$. Notice that for each function evaluation the $\mathrm{k}-\mathrm{d}$ tree has to be reconstructed since using different weights $w_{i}$ for the shape parameters results in a different point distribution.

The defined function $f$ is a nonlinear function depending on $6+t$ parameters. To minimize $f$ an algorithm is used which combines a simulated annealing technique with the downhill simplex algorithm by Nelder and Mead [PFTV92] in order to find a near global minimum. This requires an initial guess for the rigid-body transformation, which is obtained by rigid points to surface registration, using the Iterative Closest Point (ICP) algorithm [BM92]. To decrease the search space, bounds to the parameters are applied.

\section{Results}

\subsection{Simulation}

Ten right femurs have been used to build the statistical shape model and experiments have been done with the remaining femur, on which 60 points were interactively selected to simulate a realistic intra-operative digitization.

Table 1(a) shows the relative importance of the modes of variation for the knee model. It can be seen from this table that the first four modes already represent more than $90 \%$ of the shape variation in the model. Mode 1 , which accounts for almost $70 \%$ of the total variance within the model, can be seen to 
be primarily concerned with describing global scaling. Since the iteratively computed mean shape is only an approximation, the 10th eigenvalue is not exactly 0.0 but it is relatively low. Fig.3(b) shows the effect of applying \pm 3 standard deviations of the first two modes of the obtained model to the mean shape.

After rigid alignment and optimization of the fitting function, the resulting root mean square error (RMS) between the test data and the registered model as well as the RMS between the complete test shape and the registered model are computed. As expected the final RMS between the test data and the registered model decreases using more deformation modes $(\sim 0.7 \mathrm{~mm}$ using one mode to $\sim$ $0.4 m m$ using 5 modes) while this is not always the case for the RMS between the test shape and the registered model. Similar results are obtained by permuting the model in the set of specimen. Fig.4(a) (top) shows the model (triangle mesh) and the test femur after the initial rigid registration with the ICP algorithm. Fig.4(a) (bottom) shows the model and the reference femur after the non rigid registration. The black spots represent the data points.

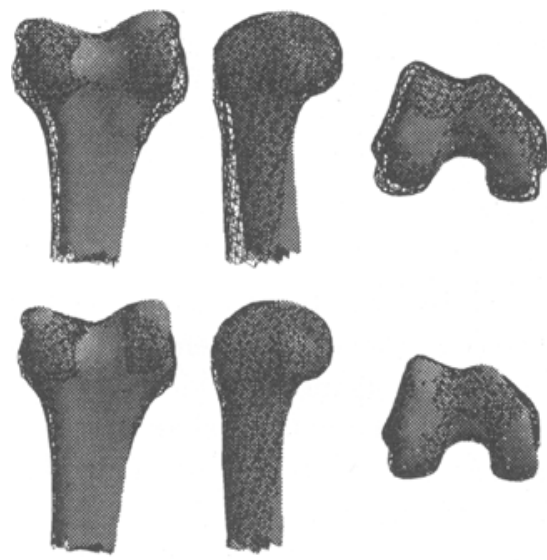

(a)

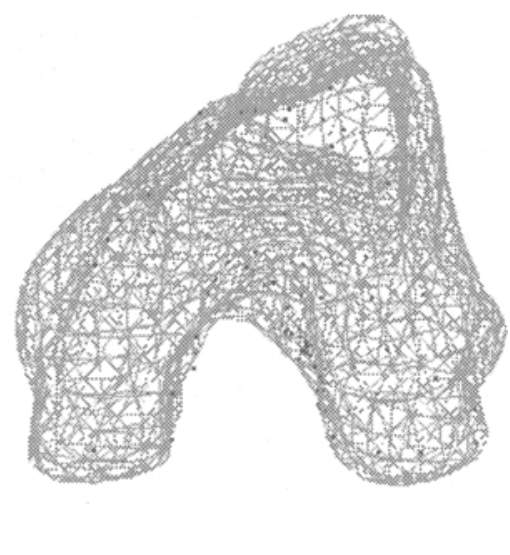

(b)

Fig. 4. (a) Simulation: the model after rigid (top) and after non rigid (bottom) registration (b) Clinical case: the model after non-rigid fitting using 6 modes in comparison with the sparse set of points.

\subsection{Experiments with real intra-operative data}

For two clinical cases, the surgeon acquired about 100 points randomly distributed on the femoral notch surface (not limiting the acquisition to a small patch and the anterior border of the notch). Results of fitting the model with those data are provided in Table 1(b). Fig.4(b) shows the deformed statistical 
model that fits the points collected during surgery. Although additional local deformation of the model would be necessary for perfect fitting, the result is satisfactory.

\begin{tabular}{|r||r|r|r|}
\hline Nr. & Eigenvalue & Percentage & Sum \\
\hline \hline 1 & 5500.59 & 69.40 & 69.40 \\
\hline 2 & 880.37 & 11.11 & 80.50 \\
\hline 3 & 548.97 & 6.93 & 87.43 \\
\hline 4 & 227.88 & 2.87 & 90.30 \\
\hline 5 & 191.34 & 2.41 & 92.72 \\
\hline 6 & 155.18 & 1.96 & 94.67 \\
\hline 7 & 128.95 & 1.63 & 96.30 \\
\hline 8 & 107.22 & 1.35 & 97.65 \\
\hline 9 & 104.08 & 1.31 & 98.97 \\
\hline 10 & 81.91 & 1.03 & 100.00 \\
\hline
\end{tabular}

(a)

\begin{tabular}{|r|r|r|}
\hline RMS error & Case 1 & Case 2 \\
\hline \hline Rigid alignment & 2.23 & 2.82 \\
\hline Model fitting with 2 modes & 2.07 & 2.17 \\
\hline Model fitting with 4 modes & 1.75 & 1.90 \\
\hline Model fitting with 6 modes & 1.61 & 1.83 \\
\hline
\end{tabular}

Table 1. (a) Relative importance of the modes of variation for the model (b) Residual fitting errors (in $\mathrm{mm}$ ) for two clinical cases using rigid alignment and using model fitting with 2,4 or 6 modes.

\section{Conclusion}

First experimental results show that the chosen approach may be successfully applied to a system for computer-assisted anterior cruciate ligament reconstruction, although it must be mentioned that 10 training shapes of which the model has been built are maybe not sufficient to represent the natural shape variation of the femur. Therefore further experiments must be done to validate the chosen approach, with special care for pathological deformations.

The current implementation requires a few minutes for fitting the statistical model with 100 data points, but optimisation of the method is possible for instance using gradient descent techniques.

The proposed method is a general technique for building specific models that can be applied to any case where the shape is stable and for which only a partial area can be digitized.

One of the key features of this approach is to use a volumetric elastic registration method (using octree-splines) to establish point correspondences between training sets which differ by their density and local distribution of points.

\section{References}

[BM92] P.J. Besl and N.D. McKay. A method for registration of 3-D shapes. IEEE Transactions on Pattern Analysis and Machine Intelligence, 14(2):239-256, 1992. 
[BV91] R.M. Bolle and B.C. Vemuri. On three-dimensional surface reconstruction methods. IEEE Trans PAMI, 13(1):1-13, 1991.

[CCMS97] A. Ciampalini, P. Cignoni, C. Montani, and R. Scopigno. Multiresolution decimation based on global error. The Visual Computer, 13(5), 1997.

[CTCG95] T.F. Cootes, C.J. Taylor, D.H. Cooper, and J. Graham. Active shape models - Their training and application. Computer Vision and Image Understanding, 61(1):38-59, 1995.

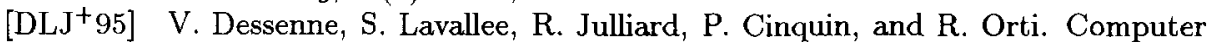
assisted knee anterior cruciate ligament reconstruction : first clinical tests. In Conference of Computer Vision, Virtual Reality, Robotics in Medicine (CVRMed'95), LNCS Series 905, pages 476-480. Springer, 1995.

[DLO+ ${ }^{+95}$ V. Dessenne, S. Lavallee, R. Orti, R. Julliard, S. Martelli, and P. Cinquin. Computer assisted knee anterior cruciate ligament reconstruction : first clinical tests. J. of Image Guided Surgery, 1(1):59-64, 1995.

[FBF77] J. H. Friedman, J.L. Bentley, and R. A. Finkel. An algorithm for finding best matches in logarithmic expected time. ACM Trans. Math. Software, 3(3):209-226, Sept. 1977.

[HDDW92] H. Hoppe, T. DeRose, T.and McDonald J. Duchamp, and Stuetzle W. Surface reconstruction from unorganized points. In Catmull E. E., editor, Computer Graphics (SIGGRAPH '92 Proceedings), pages 71-78, July 1992.

[JLD98] R. Julliard, S. Lavallee, and V. Dessenne. Computer Assisted Anterior Cruciate Ligament Reconstruction. Clinical Orthopaedics and Related Research, 1998.

[LSB91] S. Lavallee, R. Szeliski, and L. Brunie. Matching 3-D smooth surfaces with their 2-D projections using 3-D distance maps. In SPIE Vol. 1570 Geometric Methods in Computer Vision, pages 322-336, San Diego, CA, July 1991.

[MT96] T. McInerney and D. Terzopoulos. Deformable models in medical image analysis: a survey. Medical Image Analysis, 1(2):91-108, 1996.

[PFTV92] W. H. Press, B. P. Flannery, S. A. Teukolsky, and W. T. Vetterling. Numerical Recipes in C: The Art of Scientific Computing. Cambridge University Press, Cambridge, England, second edition, 1992.

[PS91] A. Pentland and S. Sclaroff. Closed-Form Solutions for Physically Based Shape Modeling and Recognition. IEEE Transactions on Pattern Analysis and Machine Intelligence, 13(7):715-729, July 1991.

[SkBG96] R. Szekely, A. kelemen, C. Brechbuler, and G. Gerig. Segmentation of 2D and 3D objects from MRI volume data using constrained elastic deformations of flexible Fourier surface models. Medical Image Analysis, 1(1):19-34, 1996.

[SL96] R. Szeliski and S. Lavallee. Matching 3-D anatomical surfaces with nonrigid deformations using octree-splines. Int. J. of Computer Vision (IJCV), (18)(2):171-186, 1996.

[STA96] G. Subsol, J.P. Thirion, and N. Ayache. Application of an automatically built 3D morphometric brain atlas: study of cerebral ventricle shape. In K.H. Hohne and R. Kikinis, editors, Visualization in Biomedical Computing (VBC'96) Proc. LNCS 1131, pages 373-382, Berlin, 1996. Springer-Verlag.

\footnotetext{
${ }^{0}$ Authors wish to thank Dr Rémi Julliard for his active collaboration to this project and the Anatomy Department of Grenoble University (Pr. JP Chirossel) for providing the specimen of femurs.
} 